\title{
State of the Art in der Therapie von Hautkrebs, Lungen- und Nierenzellkarzinom
}

In den interdisziplinären State-of-the-Art-Gesprächen des ONKO-Internetportals diskutieren Experten auf Basis aktueller Studienergebnisse neue Diagnose- und Therapiestandards sowie vielversprechende Neuentwicklungen in verschiedenen Indikationen. Ein Schwerpunkt der Gespräche liegt auf der Einordnung der Daten hinsichtlich ihrer Relevanz für die klinische Praxis. Updates gibt es nun zu den Themen Hautkrebs, Lungenkarzinome sowie zum metastasierten Nierenzellkarzinom. Die Aufzeichnungen stehen als Video zum Abruf oder Download bereit.

\section{Hautkrebs: Neues zu zielgerichteten und Immuntherapien}

Im Mittelpunkt des Expertengesprächs zum Thema Hautkrebs stehen u. a. neue Langzeitdaten zur zielgerichteten und Immuntherapie des metastasierten Melanoms und die Frage nach der Relevanz bei verschiedenen Subgruppen. Ebenfalls im Fokus der Diskussion: Aktuelle Ergebnisse zur Kombinationstherapie mit zielgerichteten und immunologisch wirksamen Substanzen, die Therapie in der Adjuvanz beim Melanom sowie aktuelle Studiendaten zu epithelialen Hauttumoren. Teilnehmer der Runde sind Prof. Stephan Grabbe (Mainz), Prof. Carola Berking (München) und Prof. Ralf Gutzmer (Hannover). Die Aufzeichnung finden Sie unter www.krebsgesellschaft.de/ado2017.

\section{SCLC und NSCLC: Was bleibt, was kommt}

Während sich beim SCLC und frühen NSCLC die klinische Praxis im Vergleich zum Vorjahr kaum verändert hat, gibt es beim NSCLC im Stadium III viel Bewegung. Neuerungen in der Therapie des Lungenkarzinoms, die PD Dr. Niels Reinmuth (München), Dr. Nicolas Dickgreber (Rheine), PD Dr. Alexander Schmittel (Berlin) und Prof. Rainer Wiewrodt (Münster) vorstellen, sind u. a.: Fortschritte in der Erstlinientherapie mit Pembrolizumab, der Einsatz der Immuntherapie nach definitiver Radio-Chemotherapie im Stadium III sowie die Zulassung des PD-L1-Inhibitiors Atezolizumab. Außerdem diskutieren die Experten über den Einsatz neuer zielgerichteter Substanzen. Das Video dazu finden Sie unter www.krebsgesellschaft.de/lungenkarzinom 2017.

\section{Metastasiertes Nierenzellkarzinom: Bewegung in der Erstlinie}

Nachdem sich beim metastasierten Nierenzellkarzinom zuletzt vor allem die Zweitlinientherapie deutlich verändert hat, zeichnen sich nun auch in der Erstlinie Fortschritte ab: Zu den beiden Standardoptionen Sunitinib und Pazopanib ist kürzlich der TKI Tivozanib hinzugekommen, der gegenüber Everolimus einen PFS-Vorteil zeigen konnte. Aufgrund positiver Studiendaten im Vergleich mit Sunitinib ist zu erwarten, dass künftig auch der TKI Cabozantinib zugelassen wird - so der Expertenkonsens.

Zudem wird mit der Kombination aus dem Checkpoint-Inhibitor Nivolumab und dem CTL-A4-Antikörper Ipilimumab absehbar auch die Immuntherapie in die Erstlinie einziehen. Diese Veränderungen werfen einige Fragen auf, beispielsweise nach Entscheidungskriterien, der Sequenztherapie und der Therapiedauer - Aspekte, die von den Teilnehmern der Gesprächsrunde lebhaft diskutiert werden. Es diskutieren: Prof. Dr. Markus Kuczyk (Hannover), Prof. Dr. Lothar Bergmann (Frankfurt/Main), Prof. Dr. Kurt Miller (Berlin), Prof. Dr. Christian Doehn (Lübeck), Prof. Dr. Ulrich Keilholz (Berlin) und Prof. Dr. Jürgen Gschwend (München). Das Gespräch sowie eine schriftliche Zusammenfassung der wichtigsten Punkte finden Sie unter www.krebsgesellschaft.de/rcc2017.

Alle State-of-the-Art-Gespräche im Überblick gibt es unter www.krebsgesellschaft.de/expertengespraeche oder diesem QRCode:

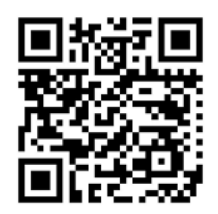

\section{Korrespondenzadresse}

\section{Dr. Pia Nitz}

Redaktion ONKO-Internetportal

In Kooperation mit der Deutschen Krebsgesellschaft e.V.

Tel. 030 - 810316112

pia.nitz@dkg-web.de 